\title{
Spitzer Surveys of IR Excesses of White Dwarfs
}

\author{
You-Hua Chu*, Robert A. Gruendl*, Jana Bilikova*, Andrew Riddle* and \\ Kate Y.-L. Su ${ }^{\dagger}$ \\ *Astronomy Department, University of Illinois \\ ${ }^{\dagger}$ Steward Observatory, University of Arizona
}

\begin{abstract}
IR excesses of white dwarfs (WDs) can be used to diagnose the presence of low-mass companions, planets, and circumstellar dust. Using different combinations of wavelengths and WD temperatures, circumstellar dust at different radial distances can be surveyed. The Spitzer Space Telescope has been used to search for IR excesses of white dwarfs. Two types of circumstellar dust disks have been found: (1) small disks around cool WDs with $T_{\text {eff }}<20,000 \mathrm{~K}$, and (1) large disks around hot WDs with $T_{\text {eff }}>100,000 \mathrm{~K}$. The small dust disks are within the Roche limit, and are commonly accepted to have originated from tidally crushed asteroids. The large dust disks, at tens of AU from the central WDs, have been suggested to be produced by increased collisions among Kuiper Belt-like objects. In this paper, we discuss Spitzer IRAC surveys of small dust disks around cool WDs, a MIPS survey of large dust disks around hot WDs, and an archival Spitzer survey of IR excesses of WDs.
\end{abstract}

Keywords: white dwarf, IR excess, circumstellar dust

PACS: 97.10.Fy

\section{INTRODUCTION}

The photospheric emission of white dwarfs (WDs) has relatively simple spectra and can be approximated by the Rayleigh-Jeans Law at infrared (IR) wavelengths. If a WD shows much higher IR fluxes than expected from its photosphere, there must be an external object contributing to the IR emission, such as a low-mass stellar or sub-stellar companion, a planet, or a circumstellar dust disk. Near-IR excesses in the $J H K$ bands have been routinely used to search for low-mass companions. Observations at longer wavelengths have been successful in finding brown dwarf companions and dust disks around WDs, but no planets around WDs have been unambiguously detected through IR excesses.

The launch of the Spitzer Space Telescope made it possible to perform imaging surveys in the 3.6, 4.5, 5.8, and $8.0 \mu \mathrm{m}$ bands with the Infrared Array Camera [IRAC, 4], and in the 24, 70, and $160 \mu \mathrm{m}$ bands with the Multiband Imaging Photometer for Spitzer [MIPS, 12]. In addition, the Infrared Spectrograph [IRS, 6] on-board Spitzer can be used to take spectra from 5 to $37 \mu \mathrm{m}$. These instruments are ideal for investigating the frequency of occurrence and physical nature of IR excesses of WDs.

We are mainly interested in IR excesses caused by circumstellar dust disks of WDs. Debris disks of stars should have dissipated well before the stars evolve off the main sequence [e.g. 11]. The circumstellar dust of WDs must have been generated recently. G29-38 and GD362 are the first two WDs reported to possess circumstellar dust disks $[1,9,11,14]$. These dust disks have been suggested to originate from tidally crushed 
asteroids, and this origin is supported by their small distances from the central WD (within the Roche limit), silicate features in the dust emission, and high metal content in the central WD's atmosphere [7, 8]. A much larger dust disk has been discovered around the central WD of the Helix planetary nebula (PN); this dust disk, extending 35-150 AU from the central WD, is suggested to be produced by collisions among Kuiper Belt-like objects [13].

These two types of dust disks can be understood in the context of stellar evolution. As an intermediate- or low-mass star evolves to the final WD stage, a large fraction of its initial mass is lost to form a PN. If a star has a planetary system, the reduced stellar gravitational field will cause the planetary system to expand. The expansion rejuvenates the internal kinematics of the planetary system. Through scattering and orbital resonances with giant planets, the sub-planetary objects may experience higher collision rates and produce dust along their orbits, and some sub-planetary objects may be scattered into the Roche limit and be tidally pulverized.

The solar system has an Asteroid Belt at $\sim 3$ AU and a Kuiper Belt at 30-50 AU from the Sun. Using the solar system analog, we expect dust around WDs to be produced within (1) the Roche limit, (2) the Asteroid Belt, and (3) the Kuiper Belt. Considering that the progenitors of WDs have a range of masses and that planetary systems around WDs have expanded from their original locations, we expect these dust disks around WDs to be detected at radial distances of (1) $<0.01 \mathrm{AU},(2)$ a few to over $10 \mathrm{AU}$, and (3) a few tens to over $100 \mathrm{AU}$, respectively.

This paper will focus on Spitzer surveys for dust disks around WDs, how they target these different types of dust disks.

\section{SPITZER WAVEBANDS AND EXPECTED DUST EMISSION}

The Spitzer Space Telescope can be used to image dust emission in the 3.6, 4.5, 5.8, 8.0, 24,70 , and $160 \mu \mathrm{m}$ bands in principle, but the poorer sensitivity and angular resolution of MIPS at 70 and $160 \mu \mathrm{m}$ make it less desirable to survey in these two bands. We will thus examine the feasibility of using the IRAC bands and the MIPS $24 \mu \mathrm{m}$ band to survey circumstellar dust disks of WDs.

The equilibrium temperature of a dust grain can be calculated by equating the absorbed stellar radiation to the radiated dust emission. Assuming a total absorption (i.e. zero albedo) and an earth radius $\left(R_{\oplus}\right)$ for the WD, the dust temperature $\left(T_{\mathrm{g}}\right)$ can be approximated by $T_{\mathrm{g}}=T_{\mathrm{WD}}\left(R_{\oplus} / 2 D\right)^{1 / 2}$, where $T_{\mathrm{WD}}$ is the effective temperature of the WD, and $D$ is the radial distance from the WD. Figure 1 plots the curves of dust temperatures as a function of $T_{\mathrm{WD}}$ and $D$. The dust temperatures are selected so that the peak emission wavelengths match the Spitzer wavebands.

The top panel of Figure 1 is optimized to illustrate dust temperatures for tidally crushed asteroids. The highest dust temperature plotted is $1500 \mathrm{~K}$, the sublimation temperature. The Roche limit is plotted in solid and dash-dot lines for asteroids of

densities 1 and $3 \mathrm{~g} \mathrm{~cm}^{-3}$, respectively. The shaded area shows the range of stellar temperatures for which the dust emission from tidally crushed asteroids can be detected in the Spitzer bands. It is clear that only WDs with effective temperatures lower than $\sim 20,000 \mathrm{~K}$ can possess such dust disks. For WDs with higher temperatures, the crushed 

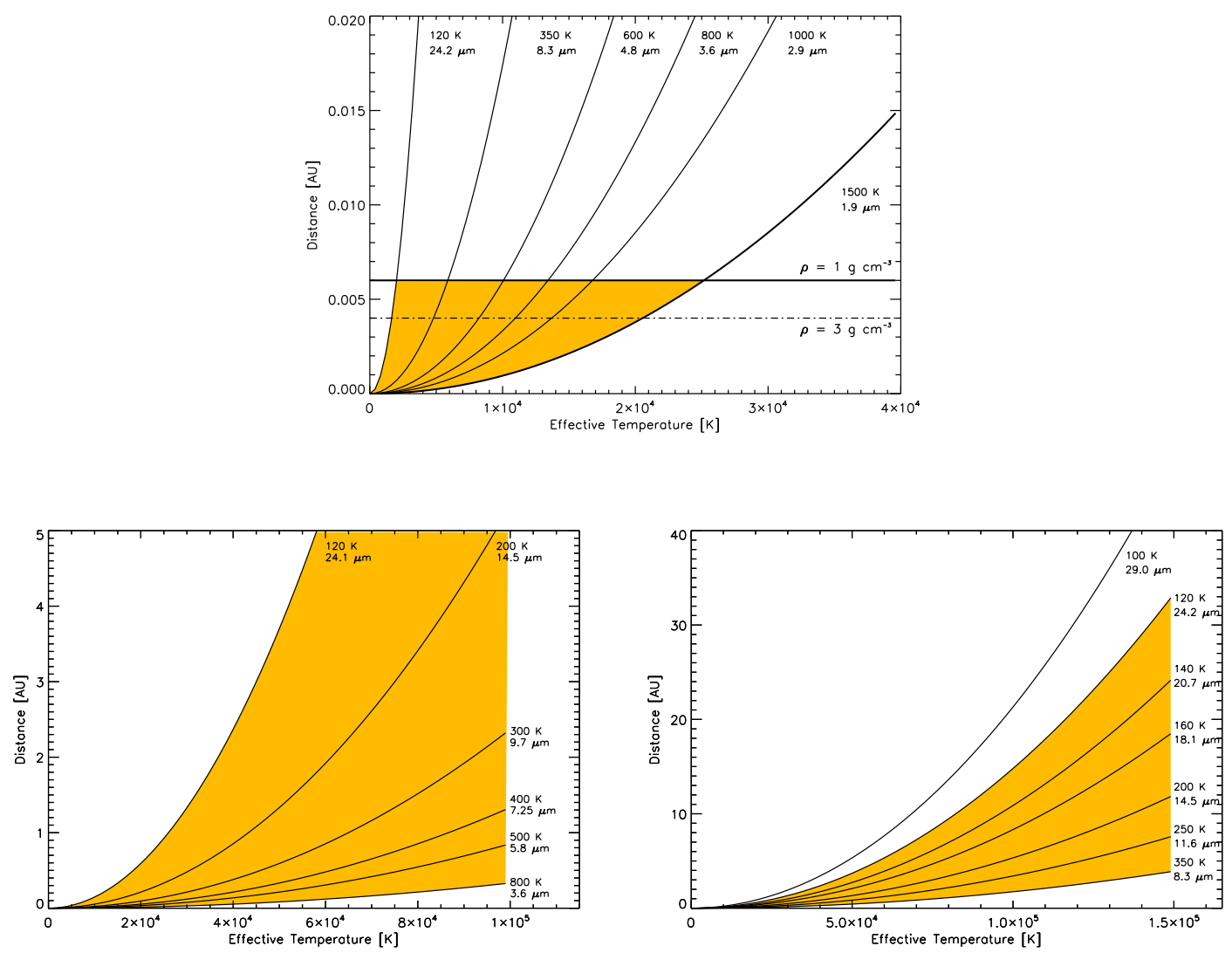

FIGURE 1. Iso-temperature curves of dust as a function of stellar effective temperature and radial distance to the central star. Some temperatures are selected so that the blackbody peak emission wavelengths are comparable to the central wavelengths of Spitzer IRAC bands and MIPS $24 \mu \mathrm{m}$ band. The three panels are optimized to show dust temperatures for three different ranges of radial distances. In the top panel, the dust sublimation temperature $(1500 \mathrm{~K})$ curve is plotted for reference, and the Roche limits for asteroids of densities 1 and $3 \mathrm{~g} \mathrm{~cm}^{-3}$ are plotted in solid and dash-dot lines, respectively. The shaded area marks the parameter space where dust emission from tidally crushed asteroids can be detected. The two lower panels show the expected dust temperatures at distances of the Asteroid Belt and Kuiper Belt, respectively.

asteroids may sublimate and form gaseous disks, such as those reported by Gänsicke et al. [5]. The Spitzer IRAC bands are ideal for surveys of such small dust disks.

The lower left panel of Figure 1 shows that at 3-5 AU from a WD, roughly where the Asteroid Belt is from the sun, the dust temperature is so low that the peak emission wavelength is much longer than those of the IRAC bands. MIPS $24 \mu \mathrm{m}$ surveys of WDs $\sim 50,000 \mathrm{~K}$ may detect dust emission at radial distances of a few AU. Emission from dust in Asteroid Belts can be surveyed at longer or shorter wavelengths for WDs of lower or higher temperatures, respectively, but not with Spitzer.

The lower right panel of Figure 1 shows that at a few tens of AU from a WD, roughly where the Kuiper Belt is located, the dust temperature is still lower. Only MIPS 24 $\mu m$ surveys for WDs with effective temperatures $\sim 100,000 \mathrm{~K}$ or hotter may detect dust emission at radial distances of a few tens of $A U$. For any cooler WDs, surveys for such cold dust need to be carried out at longer wavelengths. 


\section{SPITZER SURVEYS OF WHITE DWARFS}

Many Spitzer programs have been designed to survey WDs. Some are general surveys, and some target specific types of WDs. The entire Spitzer archive contains many observations that targeted other objects but serendipitously included WDs in the fields. Roughly four categories of Spitzer surveys of WDs exist:

- Broad survey programs by Kuchner (P2313; IRAC), Kilic (P474; IRAC), Farihi (P30807; IRAC), and Chu (P40953; MIPS). A total of 310 WDs were surveyed in these programs.

- Survey programs targeting metal-rich WDs, such as DZ, DAZ, DBZ, etc., by Jura (P275 and P50060; IRAC), Burleigh (P30432 and P60161; IRAC), Farihi (P30807, P60119, P70037, and P70116; IRAC), Debes (3655; IRAC), and Kilic (P70023; IRAC). A total of about 80-90 WDs were observed in these programs.

- Survey for WDs originating from binary mergers by Hansen (P3309; IRAC). Fourteen WDs were observed in this program.

- Spitzer archive contains serendipitous IRAC observations for $~ 330$ WDs; among these $\sim 130$ WDs are detected.

We can examine the temperatures of WDs observed by Spitzer to see what types of dust disks may be detected. Figure 2 shows histograms of temperature distributions for WDs targeted specifically in Spitzer IRAC and MIPS observations, and those that were detected serendipitously in archival data. The top panel shows that Spitzer IRAC observations were mostly made for cooler WDs. For WDs cooler than 20,000 K, IRAC observations may detect dust emission from tidally disrupted asteroids. IRAC observations of WDs hotter than 20,000 K may detect dust emission at radii of $\sim 1$ AU of higher, but no IR excesses in IRAC bands are detected for WDs hotter than 20,000 K.

The middle panel of Figure 2 shows the temperature distribution of WDs targeted by MIPS $24 \mu \mathrm{m}$ observations. Two distinct groups of WDs were observed. In the cooler group, most of the WDs were observed to search for outer boundaries of dust disks produced by tidally crushed asteroids. In the hotter group, most of the WDs were specifically targeted to search for dust in the Kuiper Belt-like distances.

The bottom panel of Figure 2 shows the temperature distribution of WDs serendipitously detected in archival Spitzer IRAC observations. Preliminary examination of their optical-to-IR spectral energy distributions (SEDs) suggests that $\sim 10 \%$ show IR excesses due to late-type companions or circumstellar dust. We are still analyzing these objects in greater detail to determine the origin of the IR excesses.

Of all Spizter surveys of WDs, the most successful in finding circumstellar dust disks are the IRAC surveys of cool, metal-rich WDs [e.g. 3, 8] and the MIPS survey of hot WDs. As we now understand that the dust created by tidally destroyed asteroids will be accreted onto the central WD and enrich its atmosphere, it is thus not surprising that IRAC surveys of metal-rich WDs have much higher success rates than broad IRAC surveys of WDs [e.g. 10]. The MIPS survey of hot WDs, discussed in the next section, also detected IR excesses for a significant fraction of the targets. These would correspond to dust emission at distances of a few tens to $100 \mathrm{AU}$, equivalent to the Kuiper Belt, from the central WD. It is worth noting that no dust disks around WDs at radial distances of 


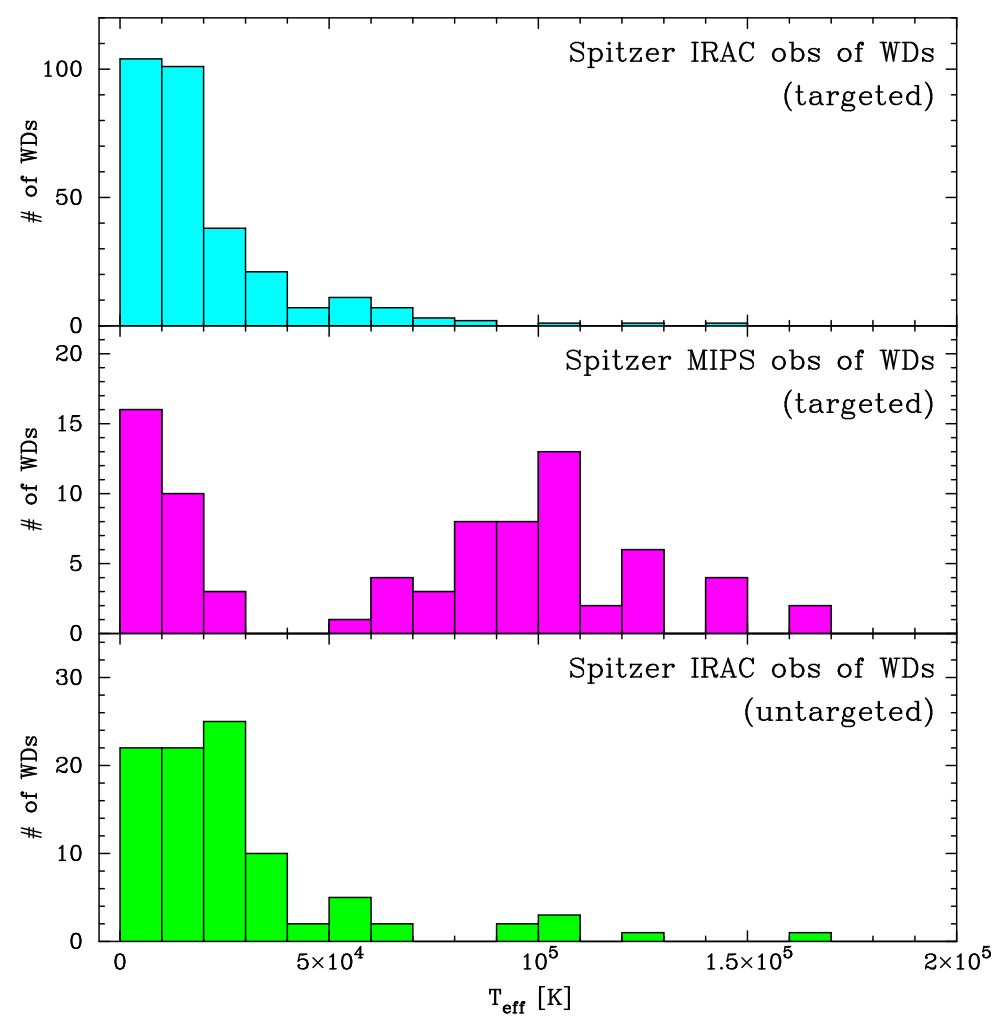

FIGURE 2. Temperature distributions of WDs observed by Spitzer in targeted IRAC and MIPS observations and WDs serendipitously detected in archival Spitzer IRAC observations.

a few AU have been detected. This is because such dust disks can be detected around WDs of temperatures $\sim 50,000 \mathrm{~K}$ using MIPS $24 \mu \mathrm{m}$ observations (lower left panel of Figure 1), but hardly any such observations were made during the cryogenic mission of Spitzer (middle panel of Figure 2).

\section{SPITZER MIPS $24 \mu$ M SURVEY OF HOT WHITE DWARFS}

Spitzer observations of the Helix Nebula revealed a bright, compact source at the central WD in the MIPS 24 and $70 \mu \mathrm{m}$ bands. Follow-up Spitzer IRS spectra of this WD confirm that the IR emission is continuum in nature and consistent with a blackbody temperature of $90-130 \mathrm{~K}$. The large emitting area, 3.8-38 $\mathrm{AU}^{2}$, can be provided only by a dust cloud. As the WD does not suffer a large extinction, the dust is likely distributed in a disk, and the disk extends 35 to $150 \mathrm{AU}$ from the central WD, of which the stellar effective temperature is $110,000 \mathrm{~K}$. As the spatial extent of this dust disk corresponds to that of the Kuiper Belt in the solar system, it is suggested that the dust disk around the central WD of the Helix Nebula was produced by collisions among Kuiper Belt-like objects [13].

Inspired by the central WD of Helix, we have carried out a MIPS $24 \mu \mathrm{m}$ survey of 71 hot WDs or pre-WDs in evolved PNe [2]. The majority of the targets have effective 
TABLE 1. Spitzer MIPS $24 \mu \mathrm{m}$ Detection of Hot WDs and Pre-WDs

\begin{tabular}{lllccc}
\hline WD Name & PN Name & WD Type & $T_{\text {eff }}(\mathrm{kK})$ & $F_{24}(\mathrm{mJy})$ & $L_{\mathrm{IR}} / L_{*}$ \\
\hline K1-22 & K1-22 & $\ldots$ & 141 & 1.07 & $3.1 \times 10^{-5}$ \\
NGC 2438 & NGC 2438 & $\ldots$ & 114 & 12.4 & $4.5 \times 10^{-4}$ \\
WD 0103+732 & EGB 1 & DA & 150 & 2.76 & $1.3 \times 10^{-5}$ \\
WD 0109+111 & $\ldots$ & DOZ & 110 & 0.27 & $4.9 \times 10^{-6}$ \\
WD 0127+581 & Sh2-188 & DAO & 102 & 0.34 & $2.7 \times 10^{-5}$ \\
WD 0439+466 & Sh2-216 & DA & 95 & 0.98 & $3.7 \times 10^{-6}$ \\
WD 0726+133 & Abell 21 & PG1159 & 130 & 0.92 & $1.6 \times 10^{-5}$ \\
WD 0950+139 & EGB 6 & DA & 110 & 11.7 & $2.6 \times 10^{-4}$ \\
WD 1342+443 & $\ldots$ & DA & 79 & 0.22 & $4.0 \times 10^{-5}$ \\
\hline WD 2226-210 & Helix Neb & DAO & 110 & 48.0 & $2.5 \times 10^{-4}$ \\
\hline
\end{tabular}

temperatures greater than $100,000 \mathrm{~K}$. Nine of these targets were detected in the $24 \mu \mathrm{m}$ observations and each represents an IR excess. These nine objects are listed in Table 1, where the Helix Nebula is also included for comparison. Follow-up Spitzer IRS spectra of K1-22, WD 0103+732, WD 0127+581, WD 0439+466, and WD 0950+139 show that in every case the emission in the $24 \mu \mathrm{m}$ band is dominated by dust continuum. The SEDs and spectral analysis are presented in the paper by Bilikova et al. in this volume.

The summary in Table 1 shows that a great majority of the hot WDs exhibiting $24 \mu \mathrm{m}$ excesses are still in PNe, and the two faintest $24 \mu \mathrm{m}$ sources belong to WDs without visible PNe. Unlike the WDs with dust disks from tidally crushed asteroids, the WDs with $24 \mu \mathrm{m}$ excesses do not necessarily show metal-enriched atmospheres, indicating that the dust accretion rates must be low.

Nine of the 71 WDs surveyed show $24 \mu \mathrm{m}$ excesses. The apparent detection rate is $12-13 \%$. We find that the WD targets without 2MASS $J$ band measurements (due to their faintness) have a lower detection rate than the brighter WD targets with 2MASS $J$ magnitudes. This is presumably a bias introduced by distance - distant WDs are fainter and detected at optical wavelengths but not in $J$ by 2MASS. Using the brighter subsample of hot WDs, we conclude that at least $15 \%$ hot WDs show $24 \mu \mathrm{m}$ excesses.

\section{REFERENCES}

1. E. E. Becklin, ApJL, 632, 119-122 (2005).

2. Y.-H. Chu, et al., AJ, to be submitted (2010).

3. J. Farihi, M. Jura, J.-E. Lee, \& B. Zuckerman, ApJ, 714, 1386-1397 (2010).

4. G. G. Fazio, et al., ApJS, 154, 10-17 (2004).

5. B. T. Gänsicke, T. R. Marsh, \& J. Southworth, MNRAS, 380, 35-39 (2007).

6. J. R. Houke, et al., ApJS, 154, 18-24 (2004).

7. M. Jura, ApJL, 584, 91-94 (2003).

8. M. Jura, et al., AJ, 133, 1927-1933 (2007).

9. M. Kilic, T. von Hippel, S. K. Leggett, \& D. E. Winget, ApJL, 632, 115-118 (2005).

10. F. Mullally, et al., ApJS, 171, 206-218 (2007).

11. G. H. Rieke, et al., ApJ, 620, 1010-1026 (2005).

12. G. H. Rieke, et al., ApJS, 154, 25-29 (2004).

13. K. Y. Su, et al., ApJL, 657, 41-45 (2007).

14. B. Zuckerman \& E. E. Becklin, Nature, 330, 138-140 (1987). 